\title{
Detection of micrometastases in peripheral blood of non-small cell lung cancer with a refined immunomagnetic nanoparticle enrichment assay
}

This article was published in the following Dove Press journal:

International Journal of Nanomedicine

28 September 2011

Number of times this article has been viewed

\author{
Qing $\mathrm{Li}^{\prime}$ \\ Hui Qi \\ Han-Xin Zhou' \\ Chun-Yan Deng' \\ Hai Zhu ${ }^{3}$ \\ Jin-Feng $\mathrm{Li}^{3}$ \\ Xi-Li Wang ${ }^{3}$ \\ Fu-Rong $\mathrm{Li}^{1,2}$ \\ 'Clinical Medical Research Center, \\ The Second Clinical Medical College \\ (Shenzhen People's Hospital), \\ Jinan University, Shenzhen, \\ People's Republic of China; \\ 2Shenzhen Institute of Gerontology, \\ Shenzhen, People's Republic of China; \\ ${ }^{3}$ Shenzhen Bioeasy Biotechnologies \\ Co Ltd, Shenzhen, People's Republic \\ of China
}

\begin{abstract}
Fe}_{3} \mathrm{O}_{4}$ particles are currently used as the core of immunomagnetic microspheres in the immunomagnetic enrichment assay of circulating tumor cells (CTCs). It is difficult to further improve the sensitivity of CTC detection or to improve tumor cell-type identification and characterization. In the present study, we prepared immunomagnetic nanoparticles with nanopure iron as the core, coated with anti-cytokeratin 7/8 (CK7/8) monoclonal antibody. These immunomagnetic nanoparticles (IMPs) were used in conjunction with immunocytochemistry (ICC) to establish a refined immunomagnetic nanoparticle enrichment assay for CTC detection in non-small cell lung cancer (NSCLC). The assay was compared with nested reverse transcription polymerase chain reaction (RT-PCR) to detect CK19 mRNA and lung specific X protein (LUNX) mRNA. Human lung adenocarcinoma cell line A549 was used for sensitivity and specificity evaluation. Peripheral blood samples were collected from each group for CTC detection. The average diameter of the immunomagnetic nanoparticles was $51 \mathrm{~nm}$, and the amount of adsorbed antibodies was $111.2 \mu \mathrm{g} / \mathrm{mg}$. We could detect down to one tumor cell in $5 \times 10^{7}$ peripheral blood mononuclear cells. The sensitivity was consistent with that of nested RT-PCR; however, the false positive rate was significantly reduced. The modified assay combined with ICC did not differ from nested RT-PCR in sensitivity, but it had significantly increased specificity. This approach could, therefore, contribute to identification of micrometastases, re-defining clinical staging, and guiding individual postoperative treatments. The technique shows considerable potential clinical value and further clinical trials are warranted.
\end{abstract}

Keywords: NSCLC, circulating tumor cells, nested RT-PCR, immunomagnetic nanoparticles, immunocytochemistry

\section{Introduction}

Lung cancer is one of the most threatening malignant tumors in humans. The overall 5 -year survival rate for all persons diagnosed with lung cancer is only $13 \%$ (47\% for focal lesion patients, $17 \%$ for regional spread patients, and $1.7 \%$ for distant metastasis patients). ${ }^{1}$ Non-small cell lung cancer (NSCLC) accounts for $80 \%$ of primary lung cancers, which are treated with surgery. However, lung cancer metastasis has already occurred in $65 \%$ of NSCLC patients at diagnosis, when it is too late for surgical treatment. ${ }^{2}$ Even when patients with stage I or II NSCLC have the opportunity to receive surgical treatment, the prognosis is poor, often with local recurrence and distant metastasis. ${ }^{3}$

Tumor metastasis is the spread of malignant tumor cells from primary lesions to secondary tissues or organs through various proliferation and growth mechanisms leading to the formation of secondary tumors. It is a multi-stage and multi-step process 
characterized by spread in the blood and lymph. Kotlyarov et $\mathrm{al}^{4}$ reported that the focal recurrence rate was $33.8 \%$, and the rate of blood-born metastasis was $55.2 \%$ during the 10 years after radical resection. Of the recurrences, $69.4 \%$ of focal recurrence and $88.7 \%$ of blood-born metastasis occurred within 1 year after surgery. ${ }^{4}$ However, no dominant metastases were found in patients undergoing preoperative or postoperative routine examinations, including surgical exploration, B ultrasound, computed tomography (CT), magnetic resonance imaging (MRI), or emission computed tomography (ECT). Therefore, micrometastasis occurring in the blood, bone marrow, lymph nodes, and other tissues and organs may be the origin of tumor recurrence and metastasis. It has been verified that the 5-year survival rate for micrometastasis patients is very low, with unfavorable prognosis. ${ }^{5}$ It is therefore of great importance for clinical diagnosis, treatment, and prognostic prediction to accurately determine the occurrence and range of micrometastasis.

CTC detection could become a valuable tool for early-stage cancer diagnosis and could serve as a real-time tumor biopsy to assess tumor invasion. Common technologies for NSCLC metastasis detection include reverse transcription polymerase chain reaction (RT-PCR) and immunocytochemistry (ICC). RT-PCR has a higher sensitivity but a higher false positive rate. ICC is regarded as having greater specificity, but it can only detect down to a sample size of $5 \times 10^{5}$ cells, although an ideal cell sample size is $2.5 \times 10^{8}$. As previously described, simple ICC technology cannot detect circulating tumor cells (CTCs) in NSCLC patients. ${ }^{6}$

Immunomagnetic nanoparticle enrichment is an advanced tool for tumor diagnosis, and immunomagnetic nanoparticles are characterized by their high specificity, ability to form high-concentration suspensions, high separation rates, and lack of influence on cell activity. It has been reported that CTC separation rates can be improved $10^{3}-10^{4}$ times by using this technology. ${ }^{7}$ In the present study, we prepared new modified immunomagnetic nanoparticles with nanopure iron as the core. We coated these nanoparticles with anti-cytokeratin 7/8 (CK7/8) monoclonal antibody and, in combination with ICC technology, we detected peripheral blood CTCs in NSCLC patients. Using this combined technology, we could isolate one tumor cell from $5 \times 10^{7}$ cells. This could contribute to the discovery of early micrometastases, monitoring of tumor recurrence and metastasis, prognosis evaluation, and preparation of individual postoperative treatments.

\section{Materials and methods Preparation of immunomagnetic nanoparticles}

Sodium alginate (100 mg, Sigma-Aldrich, St Louis, MO) was dissolved into $4 \mathrm{~mL}$ water and $2 \mathrm{~mL}$ of $5 \%$ pure $\mathrm{Fe}$ magnetic fluid solution was then added (average diameter: $10 \mathrm{~nm} \pm 5 \mathrm{~nm}$, purity: 99.99\%, specific saturation magnetization $\geq 1800 \mathrm{Am}^{2} / \mathrm{kg}$, intrinsic coercivity $\geq 34.8 \mathrm{KA} / \mathrm{m}$, provided by Shenzhen Junye Nano Material Ltd, Shenzhen, China). The solution was mixed thoroughly at $42^{\circ} \mathrm{C}$ and then underwent 15 minutes of ultrasound irradiation. $10 \% \mathrm{Na}_{2} \mathrm{CO}_{3}$ was added to adjust the $\mathrm{pH}$ to 10 , and the mixture was heated to $60^{\circ} \mathrm{C}$. Under ultrasound irradiation and high-speed mixing, the mixture was dripped into $90 \mathrm{~mL}$ di-2-ethylhexyl sulfosuccinate (AOT, Sigma-Aldrich)/ heptane oil at $60^{\circ} \mathrm{C}$ to form a transparent gray-black reverse microemulsion system. Following addition of $4.5 \mathrm{~mL} \mathrm{30 \%}$ $\mathrm{CaCl}_{2}$, the microemulsion system was subjected to immunomagnetic separation. Following washing with acetone, ethanol, and distilled water, $1500 \mathrm{rpm}$ centrifugal separation was performed and samples vacuum freeze-dried for storage.

After rinsing three times with phosphate buffer solution (PBS), $1 \mathrm{mg}$ of prepared nanoparticles was immersed in $4 \mathrm{~mL}$ of $0.01 \mathrm{~mol} / \mathrm{L} \mathrm{PBS} \mathrm{(} \mathrm{pH}=7.0)$, and $5 \mathrm{mg}$ carbodiimide (EDC, Sigma-Aldrich) and $7.5 \mathrm{mg}$ sulfo-N-hydroxysuccinimide (sulfo-NHS, Sigma-Aldrich) were added and mixed thoroughly. After 15 minutes at room temperature, $50 \mathrm{mg}$ 6-aminocaproic acid was added and the mixture was mixed for 3 hours, after which $600 \mu \mathrm{L}$ of $30 \mu \mathrm{g} / \mathrm{mL}$ cytokeratin 7/8 (CK7/8) monoclonal antibody (Chemicon, Temecula, CA) was then added and mixed for 6 hours. The mixture was then sealed with $1 \mathrm{~mL}$ of $0.2 \mathrm{~mol} / \mathrm{L}$ glycine solution containing $0.2 \%$ bovine serum albumin (BSA). The mixture was preserved at $4^{\circ} \mathrm{C}$ followed by magnetic separation and addition of storage solution. Transmission electron microscopy (TEM, TECHAI-10; Phillips, Amsterdam, the Netherlands) and light microscopy (TE2000-U; Nikon, Tokyo, Japan) were used for qualitative determination of nanoparticle morphology.

\section{Clinical specimens}

Fifty-five patients with primary NSCLC admitted to our hospital between March 2010 and May 2011 were enrolled in this study, including 30 males and 25 females, aged 43-75 years with an average age of 57.6 years. The grades were as follows: 21 stage I cases, comprising 13 squamous cell lung carcinoma cases and eight lung adenocarcinoma 
cases; 21 stage II cases, comprising 13 squamous cell lung carcinoma cases and eight lung adenocarcinoma cases; ten stage III cases, comprising six squamous cell lung carcinoma cases and four lung adenocarcinoma cases; three stage IV cases, comprising one squamous cell lung carcinoma case and two lung adenocarcinoma cases. The 55 patients were confirmed to have NSCLC after surgical inspection, and NSCLC was staged using the Tumor, Node, Metastasis (TNM) system. ${ }^{8}$ All patients were diagnosed primarily without history of other epithelial tumors and infectious diseases. Another 25 patients with benign lung diseases (BLD) were enrolled, including tuberculoma in six cases, inflammatory pseudotumor in two cases, and bronchiectasis disease in six cases. There were 14 males and 11 females. The age range was $32-67$ years, with a mean age of 53.4 years. Twenty-five healthy volunteers (13 males and 12 females with a mean age of 39.6 years, ranging 28-60 years) served as controls. There were four groups in the study: group A, stage I NSCLC; group $\mathrm{B}$, stage II-IV NSCLC; group C, benign lung disease patients; group D, healthy volunteers. All samples were collected and analyzed after obtaining written informed consent.

\section{Experimental methods}

The sensitivity of immunomagnetic nanoparticles to CTCs

Two hundred milliliters of peripheral blood were collected from healthy volunteers. Following heparin anticoagulation (BD Vacutainer; Becton Dickinson, Franklin Lakes, NJ), blood specimens were processed by density gradient separation. Peripheral blood mononuclear cells (PBMCs) were isolated and counted after rinsing twice with PBS. Human lung adenocarcinoma A549 cells were added to PBMC suspensions to give ratios of PBMCs to A549 cells of $10^{4}: 1,10^{5}: 1$, $10^{6}: 1,5 \times 10^{6}: 1,1 \times 10^{7}: 1,5 \times 10^{7}: 1$ and $1 \times 10^{8}: 1$. Twenty microliters of immunomagnetic nanoparticles were added to each specimen. The suspension was mixed for 30 minutes at room temperature, and then separated using a magnetic separator. Enriched tumor cell suspensions were coated onto slides, the slides were air-dried and detected by immunocytochemistry (ICC). The tumor cells were detected with an anticytokeratin antibody directed against cytokeratins 7 and 8, as described previously. ${ }^{9}$ Labeled cells were detected with biotinylated goat anti-rabbit IgG and streptavidin horseradish peroxidase. The streptavidin-peroxidase method was routinely used. ${ }^{9}$

The procedures described were repeated five times for each concentration. Slides were evaluated by light microscopy (40× objective), and tumor cells were evaluated by an experienced pathologist. Tumor cells with red cytoplasm were CK-positive cells. CK-positive cells were then observed under the $100 \times$ oil-immersion objective to identify nuclear specificity and nucleoli and chromatin changes to differentiate tumor cells. The tumor cell recovery rate in enriched cells was calculated. The experiment was repeated on a subsequent day. Scanning electron microscopy was also conducted.

Ten milliliters of blood were obtained from all patients. Following heparin anticoagulation (BD Vacutainer; Becton Dickinson, Franklin Lakes, NJ), the blood samples were subjected to a red cell lysis step to isolate PBMCs. The isolated PBMCs were split into two aliquots for detection of CK19 mRNA and LUNX mRNA: one using nano-immunomagnetic separation plus ICC, and simple ICC, the other using nested RT-PCR.

\section{Immunomagnetic nanoparticles enrichment assay for CTC detection}

The isolated PBMCs were placed in a $15 \mathrm{~mL}$ flask followed by the addition of $20 \mu \mathrm{L}$ immunomagnetic nanoparticles. The mixture was rotated and mixed for 30 minutes at room temperature and then separated using a magnetic separator. Enriched cell suspension samples were selected using the dropping method. CTC staining and result determination were performed using the previously described ICC method.

\section{Total RNA extraction and nested RT-PCR}

Total RNA extraction was performed according to the Trezol ${ }^{\mathrm{TM}}$ RNA Isolation Reagent kit manual (Gibco Laboratories, Lawrence, MA). We checked RNA quality by separating $5 \mu \mathrm{L}$ total RNA by electrophoresis and observing the $18 \mathrm{~S}$ and $28 \mathrm{~S}$ RNA bands. RNA was quantified spectrophotometrically using the formula: RNA $(\mu \mathrm{g} / \mu \mathrm{L})=\mathrm{A} 260 \times 40 \times$ dilution $/ 1000 . \mathrm{CK} 19 \mathrm{mRNA}$ and LUNX mRNA PCR primer sequences were designed according to the literature. ${ }^{10,11}$ Glyceraldehyde-3-phosphate dehydrogenase (GAPDH) primers were designed with DNA analysis software (Table 1). All primers were manufactured by Sangon Biotech (Shanghai, China). To test for sensitivity of nested RT-PCR, isolated PBMCs from healthy volunteers were combined with A549 cells in equal proportions for total RNA extraction. For nested RT-PCR detection, experimental procedures were performed according to the instructions of an RT-PCR kit (BioDev-Tech, Beijing, China). The RT reaction system was $20 \mu \mathrm{L}$, and reaction conditions were $37^{\circ} \mathrm{C}$ for 2 hours and $95^{\circ} \mathrm{C}$ for 5 minutes. Two rounds of PCR were performed under the same conditions: after 
Table I The sequences of CKI9, LUNX, and GAPDH primers

\begin{tabular}{|c|c|c|}
\hline Primers & Sequences of primers & Product size \\
\hline CKI9 & 5'-AAGCTAACCATGCAGAACCTCAACGACCGC-3' & \\
\hline Out primer & 5'-TTATTGGCAGGTCAGGAGAAGAGCC-3' & 1069 bp \\
\hline CKI9 & 5'-TCCCGCGACTACAGCCACTACTACACGACC-3' & \\
\hline Inner primer & 5'-CGCGACTTGATGTCCATGAGCCGCTGGTAC-3' & 745 bp \\
\hline LUNX & 5'-GGGCCTCATTGTCTTCTACGGG-3' & \\
\hline Out primer & 5'-GAATGGGTGCAGTCACCAAGGAC-3' & 534 bp \\
\hline LUNX & 5'-CTCATTGTCTTCTACGGGCTGTTAG-3' & \\
\hline Inner primer & 5'-CTTTATGCCGAGAGGGATGGT-3' & 396 bp \\
\hline \multirow[t]{2}{*}{ GAPDH } & 5'-TCCATGACAACTTTGGTATC-3' & \\
\hline & 5'-TTCAGCTCAGGGATGACCTTG-3' & 183 bp \\
\hline
\end{tabular}

Abbreviations: GAPDH, glyceraldehyde-3-phosphate dehydrogenase; LUNX, lung specific X protein; CK, cytokeratin; bp, base pairs.

denaturation at $94^{\circ} \mathrm{C}$ for 5 minutes, 35 cycles consisting of $94^{\circ} \mathrm{C}$ for 45 seconds, $58^{\circ} \mathrm{C}$ for 30 seconds, and $72^{\circ} \mathrm{C}$ for 60 seconds were performed, with a final extension step at $72^{\circ} \mathrm{C}$ for 8 minutes. Five microliters of PCR product from the second round was separated by $1.5 \%$ agarose gel electrophoresis, stained with ethidium bromide (EB), and observed using an Image Acquisition and Analysis System (Bio-Rad, Hercules, CA) with a directed image camera (Olympus, Tokyo, Japan).

\section{Statistical analysis}

SPSS software (v 13.0; IBM, Armonk, NY) was used for the statistical analysis of all data. Detection results were expressed as $\bar{\chi} \pm \mathrm{s}$. The Chi-square test was used to compare enumeration data. Results were considered to be statistically significant at $P<0.05$.

\section{Results}

\section{Morphology and diameter}

\section{of immunomagnetic nanoparticles}

When viewed under a transmission electron microscope, the immunomagnetic nanoparticles were of regular spherical shape, showed good dispersion properties and had a mean diameter of $51 \mathrm{~nm}$ (range 35-80 nm, Figure 1). Protein concentration on the immunomagnetic nanoparticles was measured by the Bradford method. One milligram of immunomagnetic nanoparticles could bind $111.2 \mu \mathrm{g}$ of antibodies. Flow cytometry showed that $98.8 \%$ of immunomagnetic nanoparticles were coated with CK $7 / 8$ antibodies.

\section{Sensitivity of immunomagnetic nanoparticles to CTCs}

The experiment was repeated five times for each concentration ratio of PBMCs to A549 cells and the results showed that no CTCs were detected at a concentration ratio of $1 \times 10^{8}: 1$;
CTCs were detected once and twice, respectively, at the ratios of $5 \times 10^{7}: 1$ and $1 \times 10^{7}: 1$. CTCs were detected in all five experiments at the ratios of $10^{4}: 1,10^{5}: 1,10^{6}: 1$, and $5 \times 10^{6}: 1$. The recovery tests, performed at two different times, gave a tumor cell recovery rate with immunomagnetic cell enrichment ranging from $86 \%$ to $93 \%$. One tumor cell in $10^{7}$ peripheral blood mononuclear cells can be detected as positive, with a specificity of $100 \%$. After immunomagnetic nanoparticle enrichment and ICC, cells with red cytoplasm were identified as tumor cells (Figure 2A). Scanning electron microscopy showed that there were numerous immunomagnetic nanoparticles present on the surface of enriched tumor cells (Figure 2B). A high magnification image shows many nanoparticles adhered to the cell membrane (Figure 2C).

\section{Detection of CTCs in NSCLC patients using immunomagnetic nanoparticle separation}

Using immunomagnetic nanoparticle enrichment and ICC, CK-positive cells were found in seven of 21 stage I NSCLC patients, while there were no CK-positive cells detected using

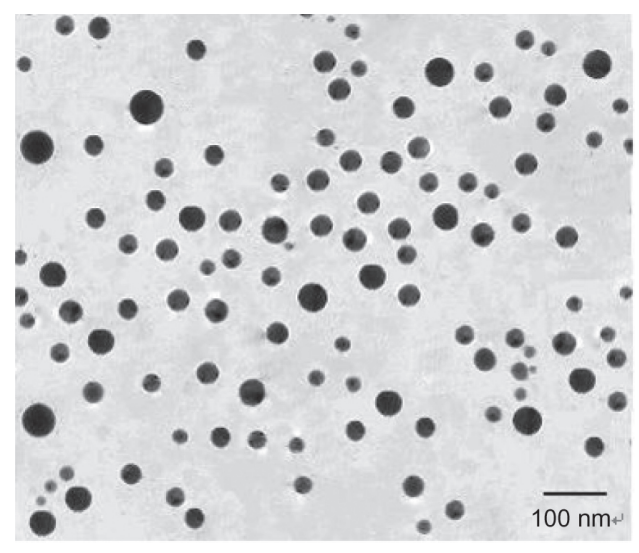

Figure I Transmission electron microscopy picture of immunomagnetic nanoparticles. 

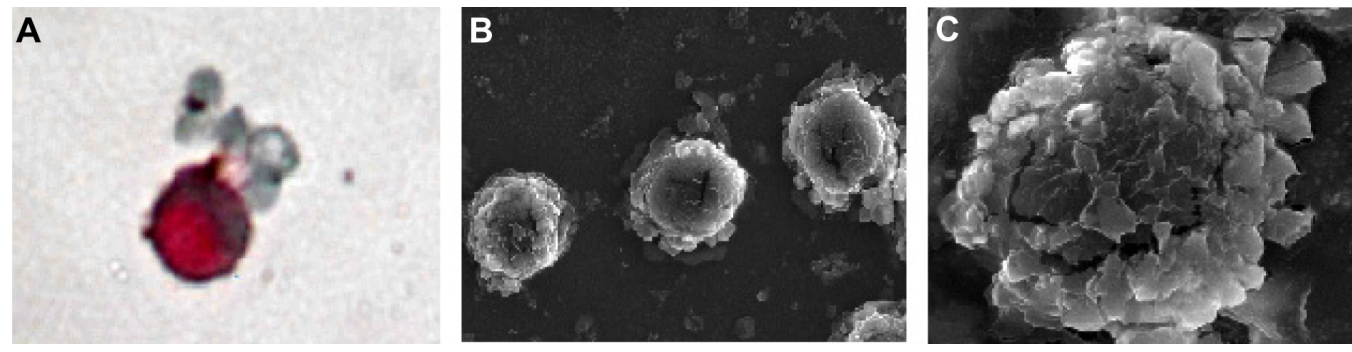

Figure 2 Images of A549 cell enrichment by immunomagnetic nanoparticles. (A) The positive enriched cell was stained with ICC; (B) SEM image of many immunomagnetic nanoparticles attached to A549 cells; (C) SEM image of immunomagnetic nanoparticles binding to the surface of an A549 cell.

Abbreviations: SEM, scanning electron microscopy; ICC, immunocytochemistry.

simple ICC. Of the 34 stage II-IV patients, CK-positive cells were detected in 22 patients by immunomagnetic nanoparticles enrichment and ICC, while, again, there were no CK-positive cells detected by simple ICC. CK-positive cells were not found in 25 benign lung disease patients and 25 healthy volunteers using immunomagnetic nanoparticle (IMP) enrichment and ICC or simple ICC (Table 2).

\section{Nested RT-PCR detection}

\section{Sensitivity results}

Following amplification using nested RT-PCR, expression of CK19 mRNA and hMAM mRNA was detected in the sample at the ratio of $1 \times 10^{7}: 1$, indicating that the lowest detectable concentration was $1 \times 10^{7}: 1$.

\section{Detection of CTCs}

Using nested RT-PCR, GAPDH was found in all 55 NSCLC patients. Of the 21 stage I NSCLC patients, CK19 mRNA expression was detected in seven patients and LUNX mRNA expression was detected in eight patients. Of the 34 stage II-IV NSCLC patients, CK19 mRNA expression was detected in 22 patients, and LUNX mRNA expression was detected in 23 patients (Figure $3 \mathrm{~A}$ and $\mathrm{B}$ ). Of the 25 benign lung patients, six patients had detectable CK19 mRNA expression and five had detectable LUNX mRNA expression. No CK19 or LUNX expression was found in the 25 healthy volunteers (Table 2).

\section{Association between the CTC positive rate and pathological features of NSCLC}

Age, sex, and smoking were not influential factors $(P>0.05)$, but pathological types of lung cancer, degree of cell differentiation, and clinical staging were significantly related to the CTC detection rate using the modified immunomagnetic nanoparticle enrichment assay $(P<0.05$, Table 3$)$.

\section{Discussion}

There is currently no valid therapy for NSCLC metastasis and postoperative recurrence. Early diagnosis of tumor micrometastasis and recurrence responses to targeted therapies can prolong the tumor-free survival period of NSCLC patients. However, the detection of tumor micrometastasis is difficult using conventional histopathology methods, leading to delays in treatment. Recent studies have established that the presence of tumor micrometastasis leads to an unfavorable prognosis in NSCLC patients. Osaki et al performed ICC testing in 115 cases of stage I NSCLC to detect reactivity to $\mathrm{AE} 1 / \mathrm{AE} 3$ anti-CK monoclonal antibodies in 2435 hilar and mediastinal lymph nodes. Univariate and multivariate analysis showed that tumor micrometastasis in lymph nodes is a marker for poor prognosis $(P=0.008$; $P=0.01) .{ }^{1}$ Yamashita et al reported that 103 patients with lung cancer micrometastases in peripheral blood had a shorter survival period than the negative group, and multivariate analysis showed that micrometastasis in peripheral blood

Table 2 Comparison of the results of three methods

\begin{tabular}{llllll}
\hline Group & $\mathbf{N}$ & ICC (\%) & IMPs + ICC (\%) & RT-PCR (\%) \\
\cline { 3 - 5 } & & & & CKI9 mRNA & LUNX mRNA \\
\hline NSCLC & 55 & 0 & $29(52.7 \%)$ & $7(33.3 \%)$ & 3 (56.4\%) \\
Stage I & 21 & 0 & $7(33.3 \%)$ & $22(64.7 \%)$ & $8(38.1 \%)$ \\
Stage II-IV & 34 & 0 & $22(64.7 \%)$ & $6(24.0 \%)$ & $23(67.6 \%)$ \\
BLD & 25 & 0 & 0 & 0 & $5(20.0 \%)$ \\
Volunteers & 25 & 0 & 0 & 0 & 0 \\
\hline
\end{tabular}

Abbreviations: NSCLC, non-small cell lung cancer; ICC, immunocytochemistry; IMPs, immunomagnetic particles; BLD, benign lung diseases; RT-PCR, reverse transcription polymerase chain reaction; LUNX, lung specific X protein; CK, cytokeratin. 

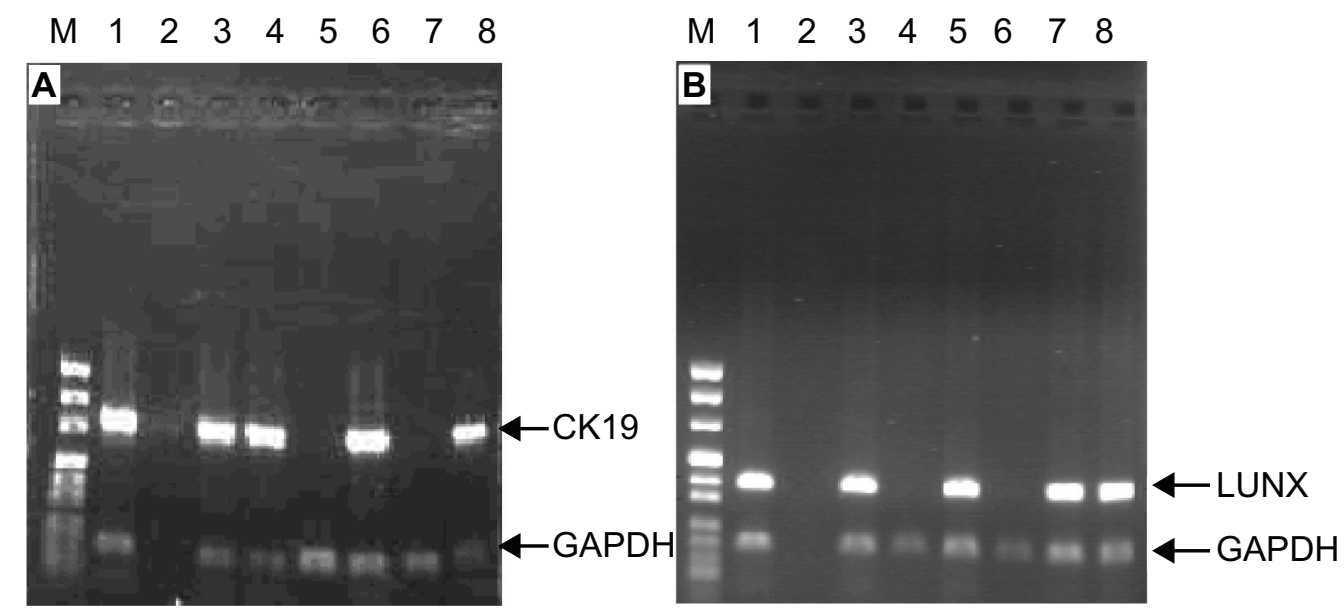

Figure 3 The expression of CKI9 mRNA and LUNX mRNA from PBMCs in NSCLC patients. (A): CKI9; (B): LUNX (M: DNA marker: Lane I: Lung cancer tissues; Lane 2: Blank control; Lanes 3-8: NSCLC patients).

Abbreviations: PBMCs, peripheral blood mononuclear cells; NSCLC, non-small cell lung cancer; GAPDH, glyceraldehyde-3-phosphate dehydrogenase; LUNX, lung specific $X$ protein; CK, cytokeratin.

before surgery is an independent prognostic indicator. ${ }^{12}$ Preparation of immunomagnetic nanoparticles and detection of micrometastasis can assist in the early detection of lung cancer at diagnosis and relapse.

Common technologies for detecting lung cancer micrometastasis include immunohistochemistry, flow cytometry, and RT-PCR. ${ }^{13}$ The immunomagnetic nanoparticle enrichment technique used to capture CTCs can facilitate concentrating CTCs $10^{3}-10^{4}$ times. Immunomagnetic nanoparticle enrichment combined with ICC and pathology examination provides

Table 3 The relationships between IMPs + ICC positive rate and NSCLC pathology

\begin{tabular}{|c|c|c|c|c|}
\hline Clinical features & $\mathbf{N}$ & $\begin{array}{l}\text { Positive } \\
\text { data }\end{array}$ & $\begin{array}{l}\text { Positive } \\
\text { rate (\%) }\end{array}$ & $P$ value \\
\hline Age (years) & & & & $>0.05$ \\
\hline$<55$ & 14 & 7 & 50.0 & \\
\hline$\geq 55$ & 41 & 22 & 53.7 & \\
\hline Sex & & & & $>0.05$ \\
\hline Male & 30 & 17 & 56.7 & \\
\hline Female & 25 & 12 & 48.0 & \\
\hline Smoking histories & & & & $>0.05$ \\
\hline Yes & 31 & 19 & 61.3 & \\
\hline No & 24 & 10 & 41.7 & \\
\hline Pathological type & & & & $<0.05$ \\
\hline Adenocarcinoma & 22 & 9 & 40.9 & \\
\hline Squamous carcinoma & 33 & 20 & 60.6 & \\
\hline Polarization degree & & & & $<0.05$ \\
\hline Poor & 21 & 16 & 76.2 & \\
\hline Well + moderate & 34 & 13 & 38.2 & \\
\hline In clinical & & & & $<0.05$ \\
\hline Stage I & 21 & 7 & 33.3 & \\
\hline Stage II-IV & 34 & 22 & 64.7 & \\
\hline
\end{tabular}

Abbreviations: NSCLC, non-small cell lung cancer; ICC, immunocytochemistry; IMPs, immunomagnetic particles. a new method for early diagnosis of NSCLC. ${ }^{14,15}$ In recent years, the continuous development of nanotechnology has seen the successful development of iron nanomaterials. We used nanopure iron microspheres as the core of immunomagnetic nanoparticles. Nanopure iron has a strong specific saturation magnetization: 2.4 times that of $\mathrm{Fe}_{3} \mathrm{O}_{4}\left(680 \mathrm{Am}^{2} / \mathrm{kg}\right)$. Combined with ICC, prepared immunomagnetic nanoparticles are superparamagnetic nanoparticles possessing high magnetic response speed, good suspension stability, high monodispersity, and a large surface area. This technology overcomes some of the deficiencies of traditional magnetic nanoparticles, such as insufficient magnetic force, slow magnetic response speed, and dissociation after cell enrichment. ${ }^{16}$

The antibody crosslinked on the surface of magnetic microspheres usually cannot achieve complete contact between the biological macromolecules and ligands as a result of steric hindrance, which reduces the space utilization of the ligands. Therefore, in this study, we linked a spacer arm of 6-amino hexanoic acid with a carbon chain length of 6 , covered with active groups, to the microspheres. Following coupling to the corresponding antibodies using EDC, high quality immunomagnetic nanoparticles were successfully prepared. ${ }^{16}$ There were up to $111.2 \mu \mathrm{g}$ antibodies per mg of immunomagnetic nanoparticles, and flow cytometry showed that $98.3 \%$ of the immunomagnetic nanoparticles possessed immunoactive antibodies. The recovery experiment showed that $93 \%$ of A549 cells could be easily and rapidly recovered using the modified immunomagnetic nanoparticles.

Using modified immunomagnetic nanoparticle enrichment and ICC methods, CTCs were detected at the ratio of $5 \times 10^{7}: 1$, indicating that the existence of a single tumor cell 
per $\mathrm{mL}$ of peripheral blood could be detected. The recovery of A597 cells was 93\%, and modified immunomagnetic nanoparticles were shown to have high sensitivity and specificity. In this study, immunomagnetic particles were combined with cytokeratin $7 / 8$ antibody, which exists in various epithelial cells and has been confirmed to be a specific marker in NSCLC. ${ }^{17}$ No positive results were obtained from the blood of 25 healthy volunteers using this technique, indicating a specificity of $100 \%$. This was an encouraging result because it established that the nanoparticle assay is specific to NSCLC cells. Compared with nested RT-PCR, the sensitivity was not different, but the false positive rate was significantly reduced.

CTCs can be detected in the peripheral blood of patients with solid tumors, and CTCs are necessary for tumor metastasis. As described above, detection of CTCs could become a standard prognostic marker. In addition, CTCs, as the "pioneers" of solid tumors, have been considered as a new target for anticancer drugs. Therefore, the detection of peripheral blood CTCs in NSCLC has important diagnostic, prognostic, and therapeutic implications. The technique shows considerable potential clinical value and further clinical trials are warranted.

\section{Acknowledgments}

The authors greatly appreciated financial support from Technology Project of Shenzhen (No. 2004A110), Technology Project of Guangdong (No. 2006B14701001), and National 863 High Technology Project (No. 2003BA310A23).

\section{Disclosure}

The authors report no conflicts of interest in this work.

\section{References}

1. Osaki T, Oyama T, Gu CD, et al. Prognostic impact of micrometastatic tumor cells in the lymph nodes and bone marrow of patients with completely resected stage I non-small-cell lung cancer. $J$ Clin Oncol. 2002;20(13):2930-2936.

2. Braun DP, Gupta D, Staren ED. Quality of life assessment as a predictor of survival in non-small cell lung cancer. BMC Cancer. 2011;11(1):353.
3. Ettinger DS, Akerley W, Bepler G, et al. Non-small cell lung cancer. J Natl Compr Canc Netw. 2010;8(7):740-801.

4. Kotlyarov EV, Rukosuyev AA. Long-term results and patterns of disease after radical operations for lung cancer. $J$ Thorac Cardiovasc Surg. 1991;102(1):24-28.

5. Mountain CF. Revisions in the international system for staging lung cancer. Chest. 1997;111(6):1710-1717.

6. Krebs MG, Hou JM, Ward TH, Blackhall FH, Dive C. Circulating tumour cells: their utility in cancer management and predicting outcomes. Ther Adv Med Oncol. 2010;2(6):351-365.

7. Martin VM, Siewert C, Scharl A, et al. Immunomagnetic enrichment of disseminated epithelial tumor cells from peripheral blood by MACS. Exp Hematol. 1998;26(3):252-264.

8. Mountain CF, Dresler CM. Regional lymph node classification for lung cancer staging. Chest. 1997;111(6):1718-1723.

9. Blümke K, Bilkenroth $U$, Schmidt U, et al. Detection of circulating tumor cells from renal carcinoma patients: experiences of a two-center study. Oncol Rep. 2005;14(4):895-899.

10. Peck K, Sher YP, Shih JY, Roffler SR, Wu CW, Yang PC. Detection and quantitation of circulating cancer cells in the peripheral blood of lung cancer patients. Cancer Res. 1998;58(13):2761-2765.

11. Mitas M, Hoover L, Silvestri G, et al. Lunx is a superior molecular marker for detection of non-small lung cancer cells in peripheral blood. J Mol Diagn. 2003;5(4):237-242.

12. Yamashita J, Matsuo A, Kurusu Y, Saishoji T, Hayashi N, Ogawa M. Preoperative evidence of circulating tumor cells by means of reverse transcriptase-polymerase chain reaction for carcinoembryonic antigen messenger RNA is an independent predictor of survival in non-small cell lung cancer: a prospective study. J Thorac Cardiovasc Surg. 2002; 124(2):299-305.

13. Zieglschmid V, Hollmann C, Bocher O. Detection of disseminated tumor cells in peripheral blood. Crit Rev Clin Lab Sci. 2005;42(2):155-196.

14. Campos M, Prior C, Warleta F, et al. Phenotypic and genetic characterization of circulating tumor cells by combining immunomagnetic selection and FICTION techniques. J Histochem Cytochem. 2008; 56(7):667-675.

15. Allan AL, Keeney M. Circulating tumor cell analysis: technical and statistical considerations for application to the clinic. J Oncol. 2010; 2010:426218.

16. Kong XL, Qi H, Zhou HX, Ren LL, Deng CY, Li FR. A novel sensitive immunoassay by nucleic acid barcode dot and its application in the detection of prostate-specific antigen. Clin Chem Lab Med. 2010;48(2): 279-283.

17. Weihrauch MR, Skibowski E, Draube A, et al. Immunomagnetic enrichment and detection of isolated tumor cells in bone marrow of patients with epithelial malignancies. Clin Exp Metastasis. 2002;19(7): 617-621.
International Journal of Nanomedicine

\section{Publish your work in this journal}

The International Journal of Nanomedicine is an international, peerreviewed journal focusing on the application of nanotechnology in diagnostics, therapeutics, and drug delivery systems throughout the biomedical field. This journal is indexed on PubMed Central, MedLine, CAS, SciSearch ${ }^{\circledR}$, Current Contents ${ }^{\circledR} /$ Clinical Medicine,

\section{Dovepress}

Journal Citation Reports/Science Edition, EMBase, Scopus and the Elsevier Bibliographic databases. The manuscript management system is completely online and includes a very quick and fair peer-review system, which is all easy to use. Visit http://www.dovepress.com/ testimonials.php to read real quotes from published authors. 